\title{
LOS Displacements of Mauna Loa volcano, Hawaii Island, as determined using SBAS-InSAR
}

\author{
Karolina Grzesiak ${ }^{1}$, and Wojciech J. Milczarek ${ }^{1, *}$ \\ ${ }^{1}$ Wrocław University of Science and Technology, Faculty of Geoengineering, Mining and Geology, 27 Wyb. Wyspiańskiego St., \\ 50-370 Wrocław, Poland
}

\begin{abstract}
This paper presents an overview of the surface displacements retrieval capability of the Differential Synthetic Aperture Radar Interferometry algorithm, using Small Baseline Subset (SBAS) technique, in the context of active volcanic areas. Based on Sentinel-1A images, time series displacements fields of the Mauna Loa volcano area over a 2-year time interval (between 2015 and 2017) were received using batch processing. Based on 35 radar images, a total of 179 interferograms have been calculated. Methodology of Synthetic Aperture Radar (SAR) Sentinel-1 ESA satellite mission data processing with small base (SBAS) interferometric techniques from has been presented. The displacements calculated in the satellite's Line of Sight (LOS) have been presented graphically on maps and graphs. Application of radar interferometry methods in the case of volcanic surface activity research creates new possibilities in the area of permanent monitoring of this type of areas.
\end{abstract}

\section{Introduction}

The present shape and location of the continents is the result of tectonic plates movements over millions of years. Numerous tectonic processes that lead to morphological differences in the terrain are observed at the boundaries of the plates. One of them is volcanism, which can both create new forms and - in the case of extremely explosions - lead to the destruction of previous forms. The earthquake is a frequent phenomenon preceding the eruption of the volcano [1]. Advanced measurement techniques enable us to obtain information about the elevation shape, observe a lot of phenomena and examine the physical properties of the Earth surface. Data for this type of research is acquired direct or indirect methods. The first group includes data from direct measurements such as GNSS, levelling, tachymetric and terrestrial laser scanning, while the second group includes radar images acquired by satellites. The range of the aforementioned methods largely depends on the quality and technical requirements, the availability of data, the physical characteristics of the object under study, and time. Remote sensing is useful in monitoring difficult to reach areas where stationary testing is dangerous, impossible to execute, or too costly. Radar interferometry is used to obtain information about the topography of the study area, as well as an excellent tool for monitoring displacements of terrain. These changes are mainly due to natural phenomena such as earthquakes, volcanic activity, landslides and human activities.

This article was created during the research work within the thesis. The main objective was to investigate
Mauna Loa's volcanic activity over two years, adequate for the operation of the Sentinel - 1A satellite, to check the suitability and quality of results based on sentinel radar images. For this purpose, 35 satellite images were processed using the open source GMT5SAR [2] program, available only on the UNIX operating systems.

\section{Mauna Loa volcano - basic information}

Mauna Loa is a shield volcano located on the southwestern side of the Hawaiian Archipelago Island Hawaii and is one of the largest active volcano in the Earth (Fig. 1). Along with the other volcanoes, it is under constant observation by the USGS Research and Development Agency. Mauna Loa occupies most of the island's total area (about $5180 \mathrm{~km}^{2}$ ), while it measures $4168 \mathrm{~m}$ above the Pacific Ocean surface [3]. The last eruption was in 1984 and it has erupted 39 times since 1832. Based on research and geological analyses, it has been shown that below the Pacific plate, there are still hot spots. The manifestation of this phenomenon allowed the formation of volcanic islands and volcanoes, by collecting the lava extracted from the interior of the land. Currently, the hot spots are located directly below the central part of the Hawai'i Islands, causing the activity in Mauna Lao and Kilauea volcanoes of the five volcanos. As a result of the eruption, the volcano produces low viscosity basaltic lava (fluid and dark). The sudden drop in pressure and temperature contributes to the crystallization of the lava, the characteristic chemical composition of 'hawaiian' lava, which causes it to move over long distances, thus forming the outer crust of a

*Corresponding author: wojciech.milczarek@pwr.edu.pl 


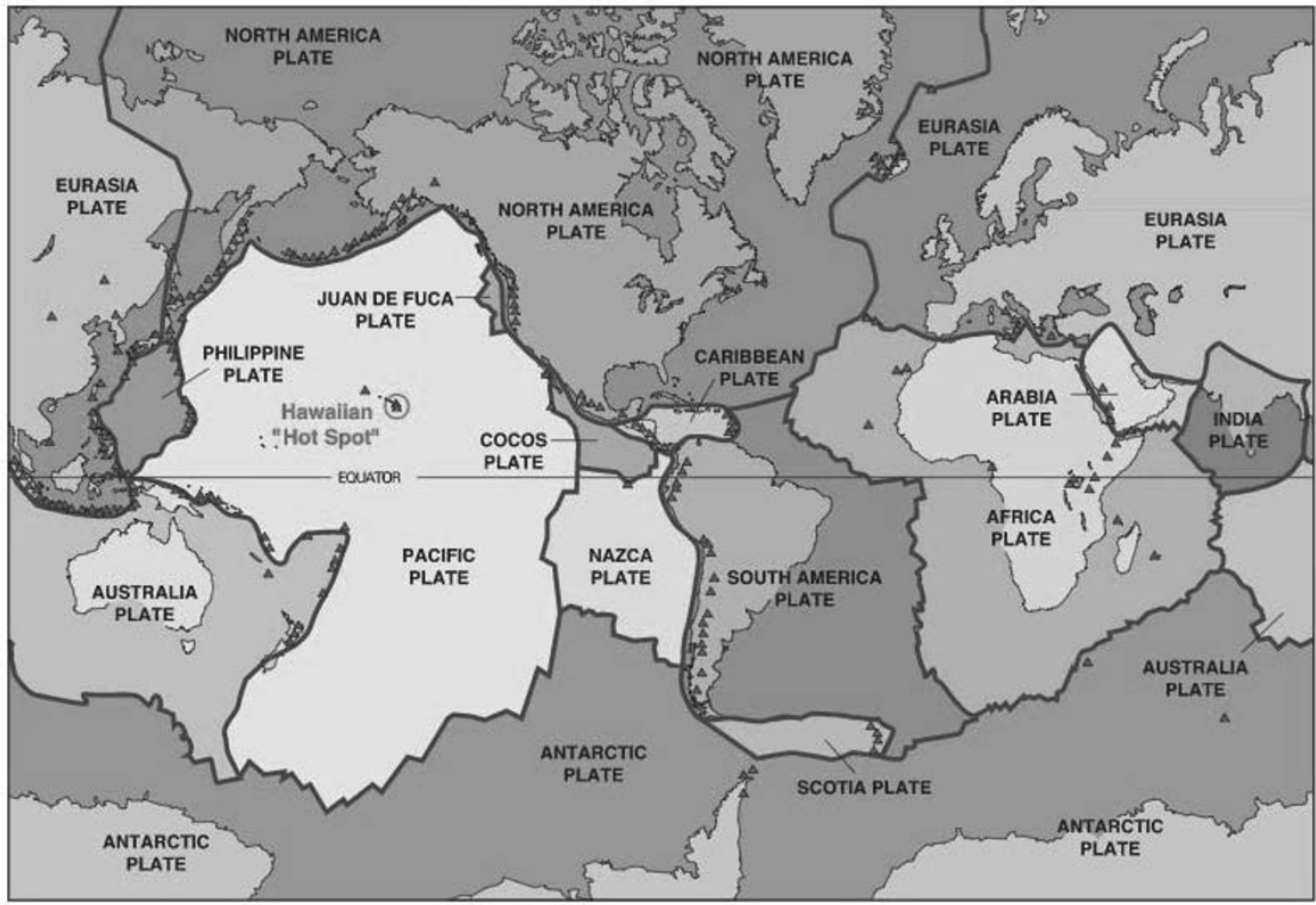

Fig. 1. The map of volcanic phenomena on global tectonics background [4].

volcano. Mauna Loa exhibits its activity more or less effectively from the dawn of history [4]. Based on numerous observations, three basic volcanic tidal zones have been distinguished for their range. The most frequent eruptions and lava flows occurred in Northeast Rift Zone and Southwest Rift Zone [5]. Various measuring techniques are used to observe volcanic activity, which can be used to the detect changes that precede volcanic eruptions. The monitoring of the volcanic area involves continuous or real - time observations, such as earthquakes, ground movements, volcanic gases. Observation is carried out using surveying instruments, GSP receivers, satellite images, cameras, thermal imaging cameras and seismic sensors. Collecting and interpreting information from different measurement techniques leads to an effective and consistent outcome for the changes in the object. Areas of the earthquake are mainly caused by eruptions within active volcanoes or deep structural changes due to the mass of islands on the earth's crust. Based on research, the HVO has shown that the vast majority of earthquakes occur in the southern part of the island, where Mauna Loa and Kalauea volcanoes are located. These quakes are divided into three classes resulting from [6]:

- the movement of magma inside volcanoes, eruptions on peaks or rift zones and the accumulation of magma in shallow reservoirs,

- plate movement within the tectonic faults,

- cambered or folded earth crust and upper mantle.

\section{Data}

Previously acquainted with the technique of generating and determining displacements, it was possible to select the appropriate input data. Data is divided into three groups: satellite images, DEM (Digital Elevation Model): the Shuttle Radar Topography Mission (SRTM) version 1 [7] and precise orbit ephemerides. Satellite images provided by the European Space Agency (ESA) were used to analyse the volcanic activity. High quality data is available on the website, which is updated on a regular basis. The data set consisted of 35 images (with perpendicular baselines of less than about $70 \mathrm{~m}$ ) captured by Sentinel - 1A satellite, moving in ascending orbits number 124 (relative orbit) between May of 2015 and June of 2017 (Tab. 1). The interval between registrations was mostly 12 days, but in occasional cases it was necessary to skip images - images didn't represent the whole object. Due to the presence of vegetation cover (Forest Reserves) Sentinel - 1A data are used, which operated on the $C$ band. Sentinel -1 potentially operates in four exclusive acquisition modes: stripmap (SM), interferometric wide swath (IW), extra-wide swath (EW) and wave (WV). SAR instrument supports operation in single and dual polarisation. For ground displacement measurements, registration in IW mode is used - so each satellite images consists of three sub swaths that have been processed as SLC products. IW mode acquires data with a $250 \mathrm{~km}$ swath at $5 \mathrm{~m}$ by $20 \mathrm{~m}$ spatial resolution. The analysed area is located in the central sub - swaths of IW2 (Fig. 2). 
Table 1. Sentinel - 1A SAR acquisitions on ascending orbits and images parameters.

\begin{tabular}{|c|c|c|c|c|c|c|}
\hline Mission & Date of registration & Orbit & Resolution & Polarisation & Mode & Sub-swath \\
\hline S1A & $30 / 05 / 2015$ & 6146 & SLC & VV & IW & IW2 \\
\hline S1A & $11 / 06 / 2015$ & 6321 & SLC & VV & IW & IW2 \\
\hline S1A & $23 / 06 / 2015$ & 6496 & SLC & $\mathrm{VV}$ & IW & IW2 \\
\hline S1A & $17 / 07 / 2015$ & 6846 & SLC & VV & IW & IW2 \\
\hline S1A & $29 / 07 / 2015$ & 7021 & SLC & $\mathrm{VV}$ & IW & IW2 \\
\hline S1A & $22 / 08 / 2015$ & 7371 & SLC & VV & IW & IW2 \\
\hline S1A & 03/09/2015 & 7546 & SLC & VV & IW & IW2 \\
\hline S1A & $15 / 09 / 2015$ & 7721 & SLC & $\mathrm{VV}$ & IW & IW2 \\
\hline S1A & 09/10/2015 & 8071 & SLC & VV & IW & IW2 \\
\hline S1A & $21 / 10 / 2015$ & 8246 & SLC & $\mathrm{VV}$ & IW & IW2 \\
\hline S1A & $25 / 01 / 2016$ & 9646 & SLC & VV & IW & IW2 \\
\hline S1A & $18 / 02 / 2016$ & 9996 & SLC & VV & IW & IW2 \\
\hline S1A & $01 / 03 / 2016$ & 10171 & SLC & $\mathrm{VV}$ & IW & IW2 \\
\hline S1A & $25 / 03 / 2016$ & 10521 & SLC & $\mathrm{VV}$ & IW & IW2 \\
\hline S1A & $12 / 05 / 2016$ & 11221 & SLC & $\mathrm{VV}$ & IW & IW2 \\
\hline S1A & $24 / 05 / 2016$ & 11396 & SLC & $\mathrm{VV}$ & IW & IW2 \\
\hline S1A & $05 / 06 / 2016$ & 11571 & SLC & $\mathrm{VV}$ & IW & IW2 \\
\hline S1A & $29 / 06 / 2016$ & 11921 & SLC & $\mathrm{VV}$ & IW & IW2 \\
\hline S1A & $11 / 07 / 2016$ & 12096 & SLC & $\mathrm{VV}$ & IW & IW2 \\
\hline S1A & $23 / 07 / 2016$ & 12271 & SLC & VV & IW & IW2 \\
\hline S1A & 04/08/2016 & 12446 & SLC & VV & IW & IW2 \\
\hline S1A & $16 / 08 / 2016$ & 12621 & SLC & $\mathrm{VV}$ & IW & IW2 \\
\hline $\mathrm{S} 1 \mathrm{~A}$ & 09/09/2016 & 12971 & SLC & $\mathrm{VV}$ & IW & IW2 \\
\hline S1A & $15 / 10 / 2016$ & 13496 & SLC & $\mathrm{VV}$ & IW & IW2 \\
\hline S1A & $27 / 10 / 2016$ & 13671 & SLC & VV & IW & IW2 \\
\hline S1A & $08 / 11 / 2016$ & 13846 & SLC & $\mathrm{VV}$ & IW & IW2 \\
\hline S1A & $02 / 12 / 2016$ & 14196 & SLC & $\mathrm{VV}$ & IW & IW2 \\
\hline S1A & $26 / 12 / 2016$ & 14546 & SLC & $\mathrm{VV}$ & IW & IW2 \\
\hline S1A & $07 / 01 / 2017$ & 14721 & SLC & $\mathrm{VV}$ & IW & IW2 \\
\hline $\mathrm{S} 1 \mathrm{~A}$ & $31 / 01 / 2017$ & 15071 & SLC & $\mathrm{VV}$ & IW & IW2 \\
\hline S1A & $12 / 02 / 2017$ & 15246 & SLC & VV & IW & IW2 \\
\hline S1A & $24 / 02 / 2017$ & 15421 & SLC & VV & IW & IW2 \\
\hline S1A & $08 / 03 / 2017$ & 15596 & SLC & $\mathrm{VV}$ & IW & IW2 \\
\hline S1A & $20 / 03 / 2017$ & 15771 & SLC & $\mathrm{VV}$ & IW & IW2 \\
\hline S1A & $01 / 04 / 2017$ & 15946 & SLC & VV & IW & IW2 \\
\hline
\end{tabular}




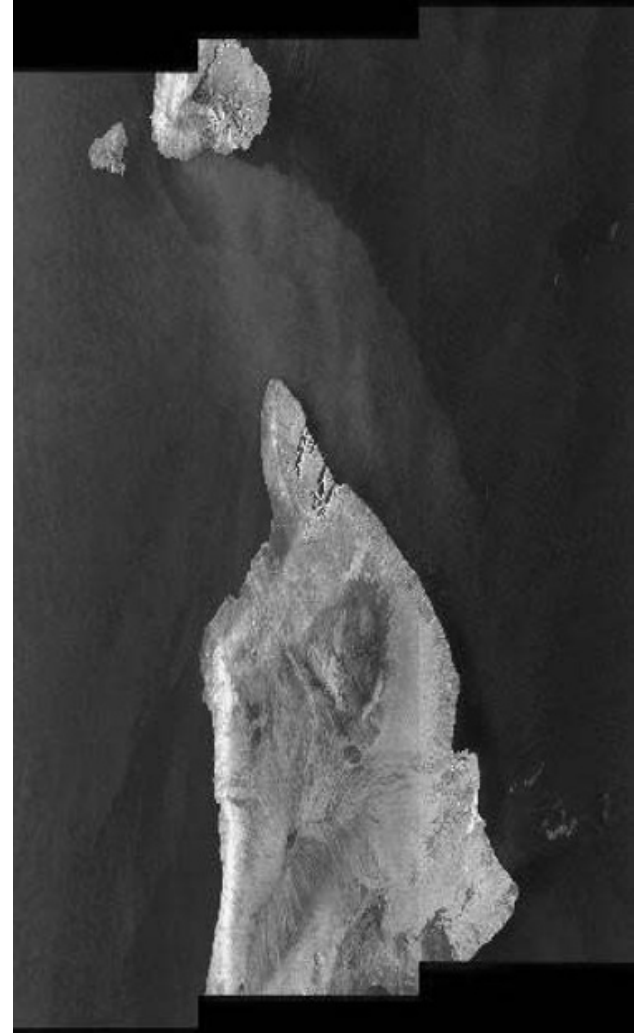

Fig. 2. Sentinel 1A image example from Hawaii Island with sub - swaths.

Another essential input file is the Digital Elevation Model - a digital model or a graphical representation of the topographic surface of the ground (Fig. 3). The model is characterized by a high resolution of $30 \mathrm{~m}$ and homogeneity in accuracy.

To generate satellite image products, the processor requires additional information not included in the satellite data. Therefore, it was necessary to retrieve auxiliary data from the PDGS - precise orbits ephemerides. The files should match the previously downloaded images for the time information.

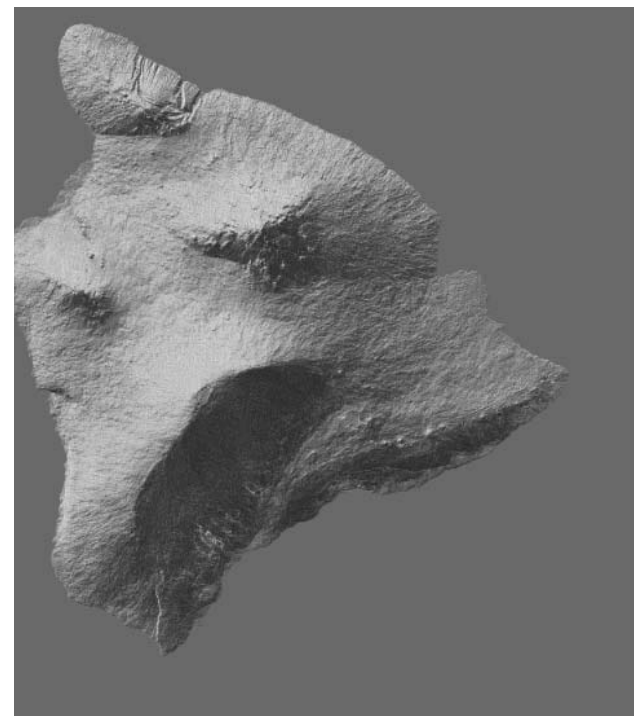

Fig. 3. Digital Elevation Models for Hawaii Island - SRTM 1.

\section{InSAR - how does it work}

InSAR method is used to measure changes in the morphology of the Earth's surface using satellite images. Information about ground changes in time interval, is included in images composed of interference bands. Based on two radar images, the phase difference for each pixel is determined, thus creating an interferogram. The selected radar images between the designated displacements should represent the same area but differ in the date registration. Determining the phase difference, attention should be paid to reflective properties of the surface, which in each image should assume equal value. Phase difference values can be obtained in single pass or repeat pass interferometry. The essential difference between modes is the number of necessary radar transits needed to obtain images. Repeat pass interferometry is a method of data registration on a single satellite only during two independent flights. Therefore, it is necessary to obtain accurate information about localization of the satellite during the registration. Based on them a spatial baseline is defined (perpendicular baseline and parallel baseline). Exceeding the critical value of the baseline makes it impossible to generate interferograms based on the selected images. Critical values depend on radar wavelength, beam angle, horizontal resolution and distance between radar and surface. The adjusted parameters determine the geometric dependence of the position of the satellites during the imaging process. Analysis of displacements and ground changes is based on the displacements phase determined, so the effect of other factors should be removed (for example topography, ground movements, atmospheric conditions, spatial distances between satellites. Elimination of the topography component can be achieved by using the DEM model. The model is converted into a synthesized interferogram. The source of interference in InSAR analyses is atmospheric artifacts and spatial decorrelation. The atmospheric artifacts are highly correlated in space but poorly in time. The deviation of the phase value can be caused by the heterogeneity of pressure, temperature or water in the troposphere. Tropospheric artifacts are present in repeat pass interferometry products, as atmospheric conditions change over time. By contrast, temporal decorrelation is related to the occurrence of vegetation, humidity changes and abundant rainfall or snowfall. It has a significant impact on the quality of interferograms generated from time baseline radar images.

\section{Method of InSAR data processing - SBAS algorithm}

Small Baseline Subset (SBAS) technique is one of the latest algorithms used to monitoring the development of surface displacements over time. The production of time - series of the line-of-sight (LOS) displacement was proposed by Berardion [8]. The SBAS - InSAR use interferograms with a small baseline to minimize topographic errors and the effects of spatial decorrelation - it is the main advantage of SBAS interferometry. Due 
to the use of distributed scatterers points, which are more commonly identified in the natural environment, this method is used for monitoring displacements in non urbanized areas. Intermediate and final results (interferograms and time - series displacements maps) are determined by batch processing, in three stages. The advantage of this process is the ability to generate several hundred interferograms based on the large amount of SAR data. All processed and calculations were performed is GMT5SAR.

\subsection{Batch pre - processing}

The first step before processing includes organize the data. The pre - processing involves both processing without a master image (using default parameters: earth radius, Doppler centroid and near range) and processing with a master image. Command execution sequence is important - the first pre - processing generates the baseline time plot necessary to select the master image to the next step. The best master image covers the area of interest and minimizes perpendicular baselines between interferometric pairs.

\subsection{Batch processing}

All activities at this stage lead to generation of interferograms. The first step is to select pairs of images from which interferograms are created, specifying the maximum value of the temporal and spatial baseline (Fig. 4). Selection of parameters determining the time intervals and perpendicular baseline should be selected in such a way that the resulting network is consistent and that each image is connected to at least two neighbours. I selected the InSAR combinations that exhibited a mutual perpendicular baseline less than $70 \mathrm{~m}$ and corresponding time intervals were set to less than 60 days. With the assumed parameters, a list of 179 interferometric pairs was obtained.

\subsection{SBAS - derived time series displacements}

This part of the work generates time - series displacement maps (corresponding to the date radar images) and velocity maps. The data parameters were also specified: smooth sf, wavelength $w l$, incidence theta, range rng and number of unwrapped interferograms.

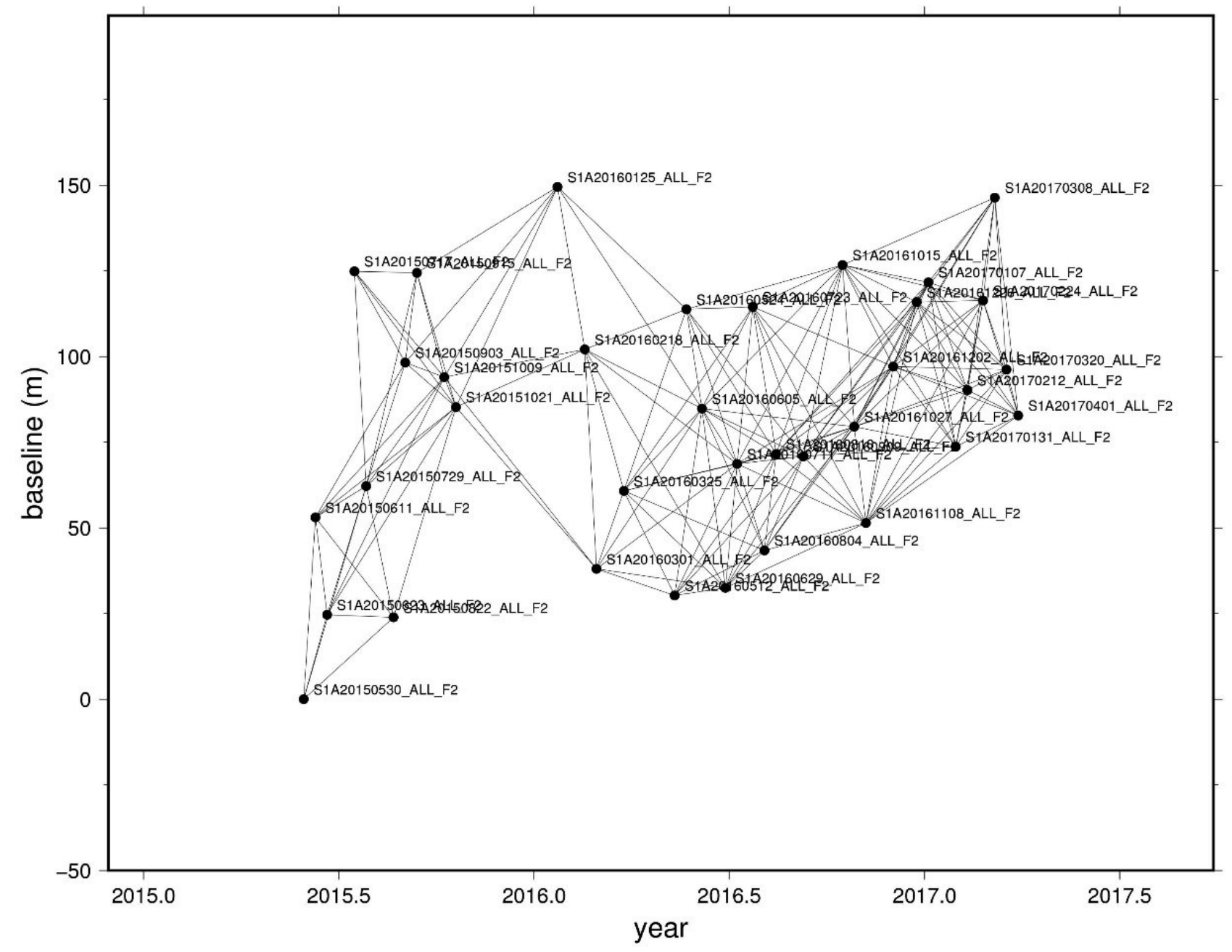

Fig. 4. Baselines and InSAR combinations. Black solid lines connection circle represents perpendicular baselines of the corresponding interferograms. 

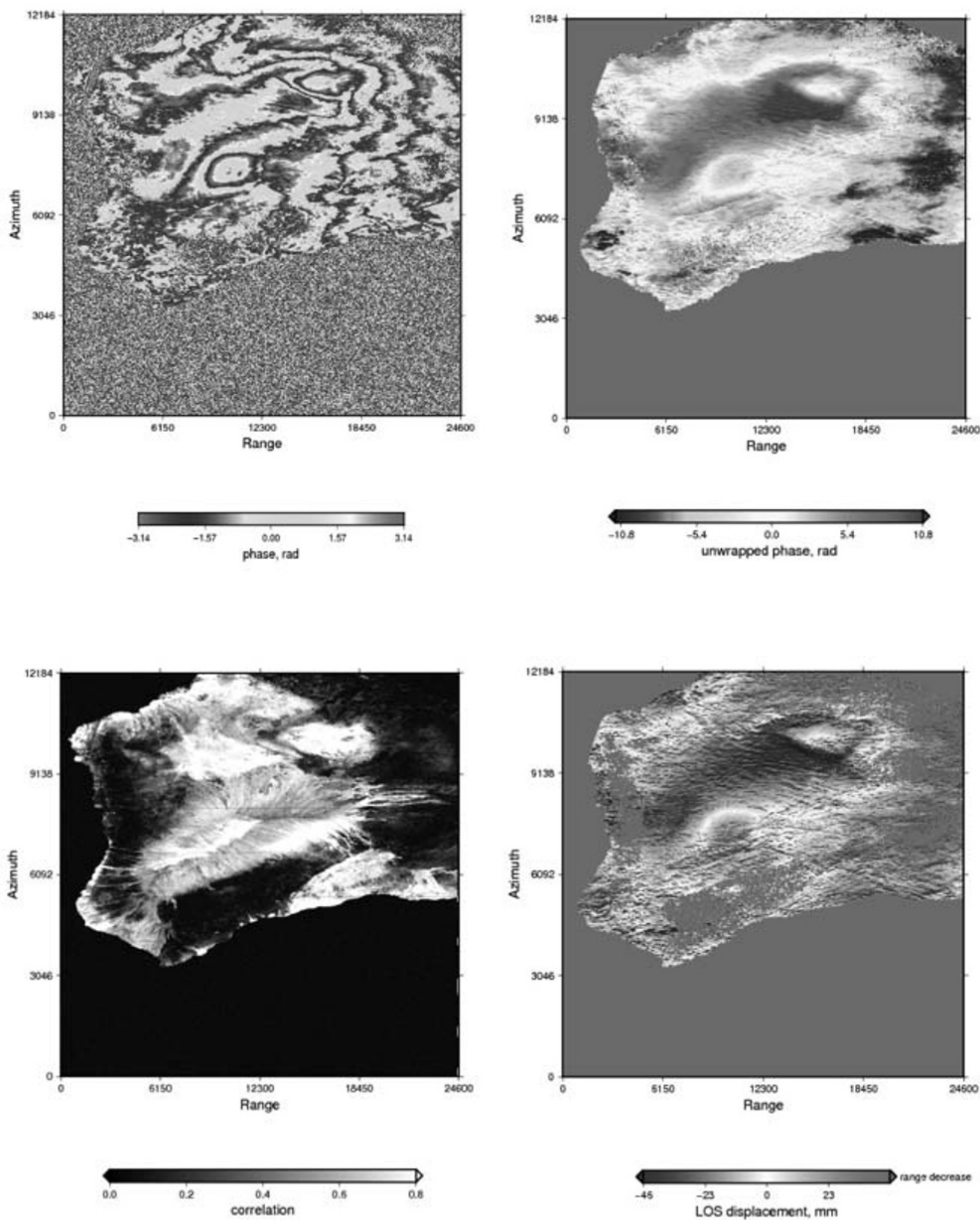

Fig. 5. Examples of calculation results for Hawaii: phase image (upper left), unwrapped phase (upper right), correlation image (lower left) and LOS displacement (lower left).

\section{Results}

The result of the process of generating interferograms for the first pair of 30.05.2015 and 11.06.2015. The use of the mask caused the elimination of water areas during the generation of interferograms. The resulting images are legible and clearly represent the study area. The correlation map includes the value of the complexity coefficient of the SAR signal, also known as the coherence map. The value is dependent on the phase interference. The value of the coefficient is in the range 
of 0 to 1 , where the pixel receiving values close to equal to 0 exhibit the phase error of the two displays, while the value equal to 1 suggests fill compliance. This example result shows both areas with low and high coherence these are mainly areas covered by vegetation (for example Forest Reserve areas) is low coherence, but the parts of the volcano surface exhibit high coherence. Based on the coherence map, the areas for which interferometric bands can be produced are determined. Within the first 12 days of observation, recorded displacement value is $4.5 \mathrm{~cm}$ and is located near the Kilauea volcano. In relation to the Mauna Loa volcano, the highest value of LOS displacements can be observed in the central part of the volcano - around the crater about $3 \mathrm{~cm}$. On the other hand, the slope of the volcano decreases. In the next step, the displacement maps were generated in time - series designated for the images input files. The results are in geographic coordinates system. Additionally, three points are identified for the analysis of volcano activity (Tab. 2).

Table 2. The coordinates of designated measurement points.

\begin{tabular}{|c|c|c|}
\hline Point & Longitude & Latitude \\
\hline P1 & -155.7805 & 19.6161 \\
\hline P2 & -155.6494 & 19.4572 \\
\hline P3 & -155.7072 & 19.2006 \\
\hline
\end{tabular}

Number one was located on the northern slope of the Mauna Loa volcano, the second was located at the western part of the cone, while the third point was on the southern flank of the volcanic edifice, due to the frequency of lava over recent years. As previously mentioned, the Island of Hawaii and volcanoes are observed by the USGS Hawaiian Volcano Observatory. According to published data of 17 September 2017, since mid-2013, seismic activity in the Mauna Loa volcano has increased compared to previous years. Prior to 2013 , on average, 15 to 20 quakes were recorded in a week, with low magnitude. However, in 2013 there was a breakthrough. Initially minor changes were observed in the frequency of the occurrence of earthquakes. By the end of 2014, the number of earthquakes rose twice during the week and thrice in 2015 - signalling changes in the earth. Based on a study of the relative distances between the GPS stations on opposite side of Mauna Loa's summit caldera, a value was changed (over 5 years period). It is often an indication of inflation of the shallow summit magma reservoir.

Figure 6 shows the time series Mauna Loa displacements fields from SBAS InSAR. To illustrate the exact displacement values, a graph of the time dependence of displacements values for the three points was created.

The displacement order increased near caldera, and the largest inflating displacement was $34 \mathrm{~cm}$ in 432 days (but the largest inflating displacement in the map range was greater than $75 \mathrm{~cm}$ in 672 days in around the
Kilauea caldera). The changes in the location of the GPS station, according to observers, is due to the magma filling of the complex tank system beneath the peak and the upper southwestern slot. Slow magma movements and filling of the tanks, under the influence of temperature and pressure, cause displacements of the layers and thus displacement of the ground. From time series displacements maps, it has been observed that from 468 days (09 September 2016) there was a decrease in the area - the lighter colours of the crater in $17 \mathrm{~g}$ and $17 \mathrm{~h}$ Figure 6. The greatest decrease was recorded in the interval of time between 576th day of measurement and 624th - it was $12.5 \mathrm{~cm}$ change. From February 2017 until the end of the measurement period, the displacement of the $\mathrm{P} 2$ point was observed again.

Displacement at point 1 oscillates within the range of $-8 \mathrm{~cm}$ to $8 \mathrm{~cm}$, thus not showing too much activity over two years. Maximum displacements between imaging registration dates are estimated at $2-3 \mathrm{~cm}$. In the first two months of research, the graph indicates a decrease in ground. By mid - 2016 measured displacement values increase, thus indicating positive displacement. These changes could have been caused by numerous earthquakes, which despite a small amount of power could have influenced the changes in terrain. Point P3 is located in the lower part of the Southwest Rift Zone. The Fig. 7 largely indicates the reduction of the area since January 2016, except for a few intervals where the value of displacement increases (e.g. Between 24th May 2016 and 5th June 2016, the highest value of displacement amounted to $7 \mathrm{~cm}$ ). In mid - February, the point was increased relative to the previous months and showed a $10 \mathrm{~cm}$ increase by the end of the period. In additional, velocity displacement maps were generated as part of the calculations. It represents mean velocity displacement exactly. Fig. 8 shows mean velocity displacement. The displacement values on the maps will be correct when the number of images in each year was approximately equal. The largest annual changes of the analysed object are observed for the Mauna Loa volcano crater, where ground displacement is increasing from 20 to $25 \mathrm{~cm}$ per year. The upper south - western slope area shows a decrease of about $10 \mathrm{~cm}$ per year, while the lower part of volcano does not show activity - it is stable. Mauna Loa volcanic surface studies were conducted by Casu [3]. From 2003 to 2008 the average annual velocity displacements near caldera was $2.5 \mathrm{~cm}$. It can be stated that volcanic activity has increased over the years. 

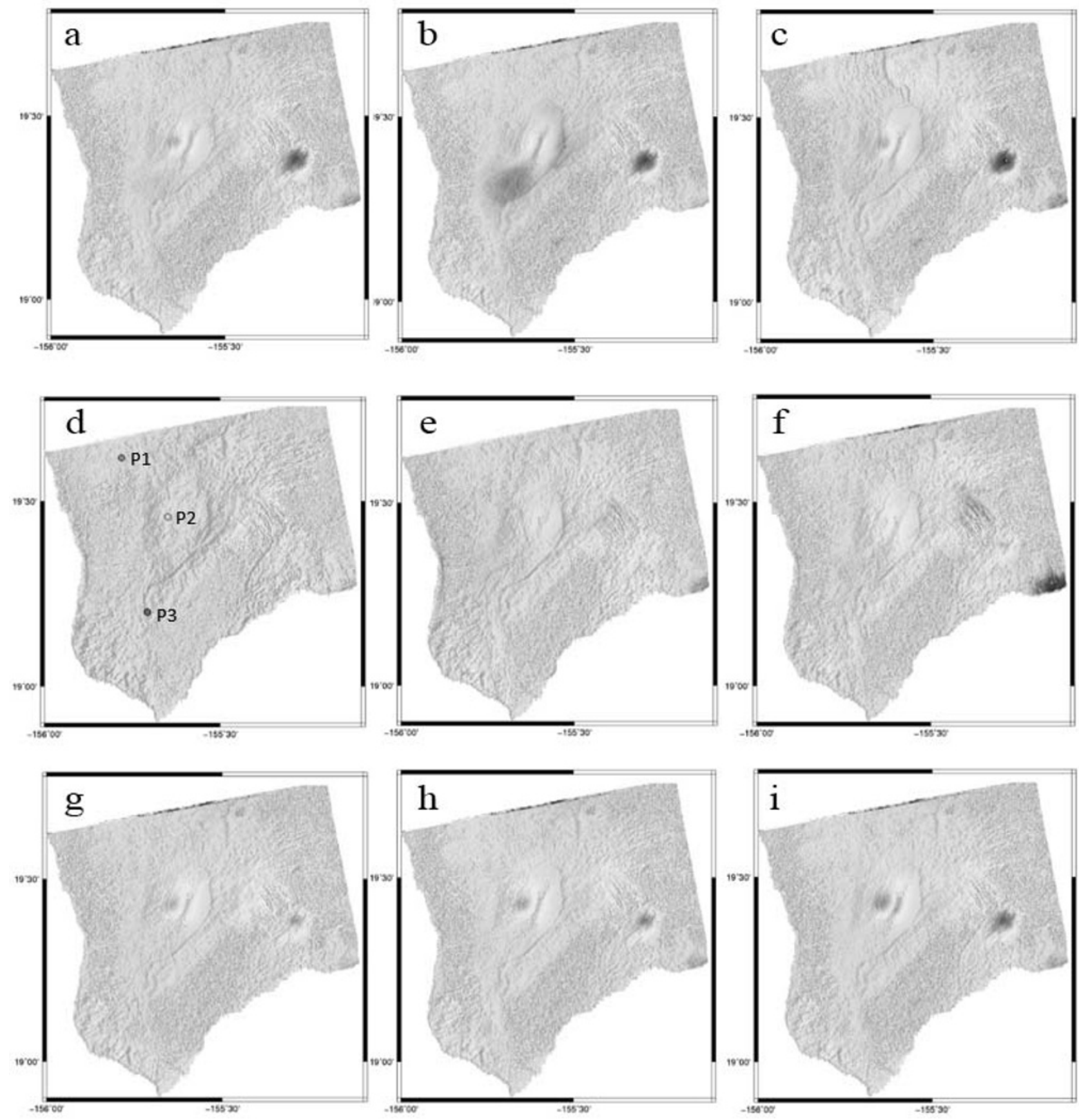

Fig. 6. Time series displacements fields derived by SBAS-InSAR (LOS) in May of 2015 and June of 2017. Accumulated displacements of (a) 24 days (b) 96 days (c) 240 days (d) 348 days (e) 408 days (f) 468 days (g) 552 days (h) 624 days (i) 672 days, are shown. 


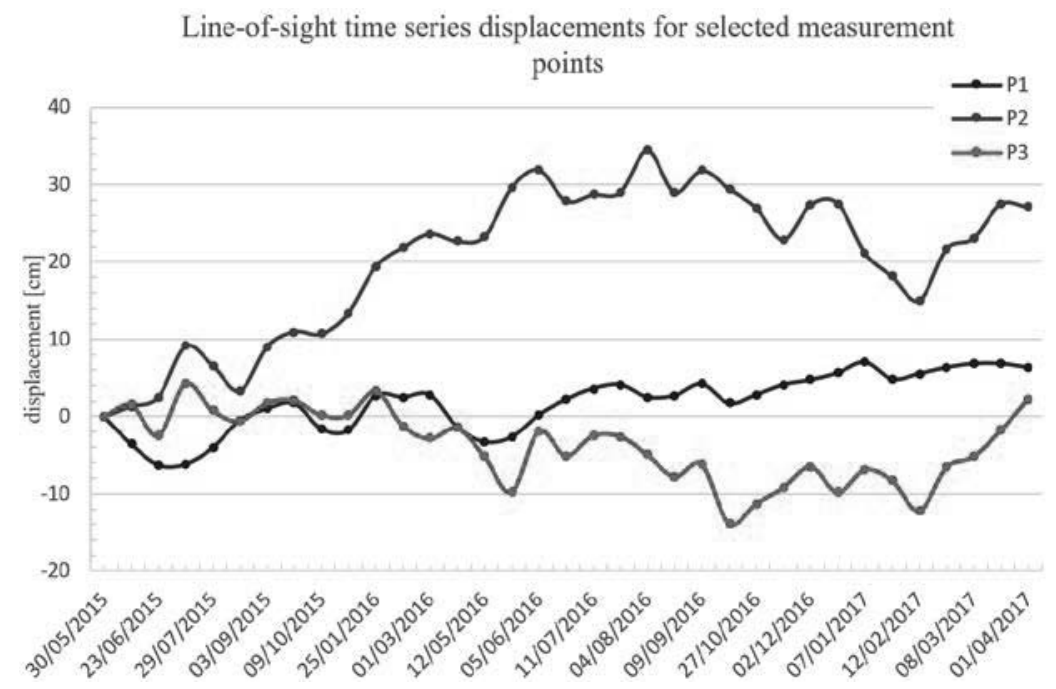

Fig. 7. InSAR LOS time series displacements for selected points.

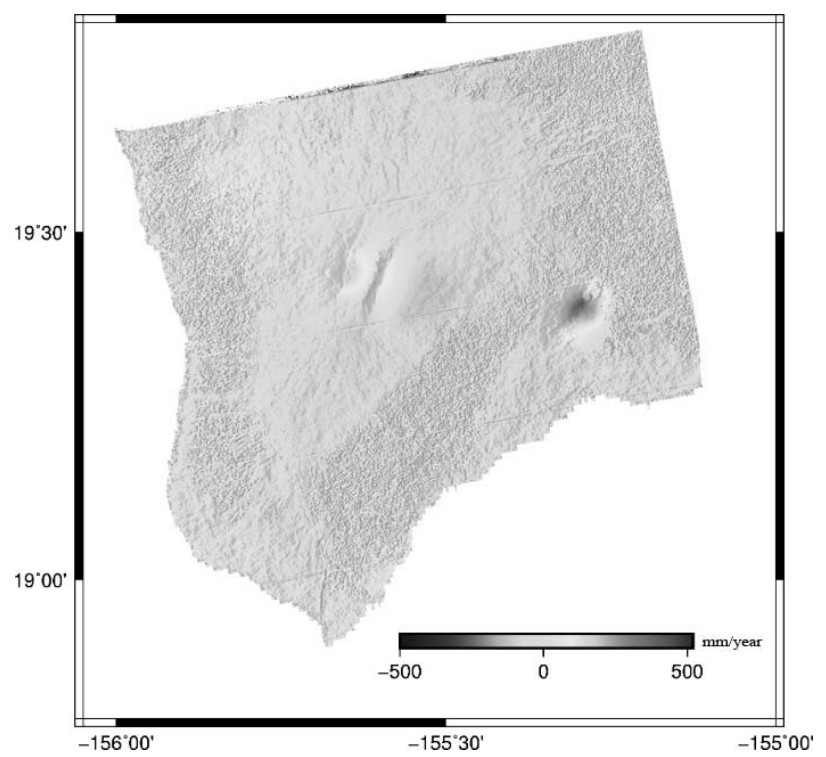

Fig. 8. InSAR LOS time series displacements for entire analysed period.

\section{Conclusions}

Thirtyfive InSAR images were used to map the displacements of the Mauna Loa volcano in 2015 2017. The displacements results showed that the Mauna Loa volcano inflated at the top of the crater. Based on the results, it can be concluded that the algorithm is efficient in determining time displacements on large non urbanized surfaces. The largest annual changes of the analyzed object are observed for the Mauna Loa volcanic crater. Based on the above results and data from the USGS, it can be said that frequent movements of Mauna Loa volcano surface result from characteristic geological processes there. The lack of eruption at the time of the study does not indicate that volcanic activity is impaired, as earthquakes can occur both on volcanic as well as on tectonic surfaces. Therefore, it is still believed that Mauna Loa is an active volcano. However, in order to obtain information regarding the exact causes of the displacement, measurements should be carried out with a variety of techniques that complement one another. The use of InSAR method in the study of volcanic surface activity is the most correct, because it generates ground displacements maps with a large spatial range of centimetres accuracy. InSAR technology allows you to track ground displacements even in bad weather conditions. Modern measurement techniques allow volcanic activity to be determined, but to be precise about the time and location of an eruption is almost impossible.

\section{References}

1. P. Migoń, Geomorfologia (Wydawnictwo Naukowe PWN, Warszawa, 2009)

2. D. Sandwell, R. Mellors, X. Tong, M. Wei, P. Wessel, Transactions American Geophysical Union, 92 (28), 234, doi:10.1029/2011EO280002 (2011)

3. F. Casu, R. Lanari, E. Sansosti, Geoscience and Remote Sensing Symposium, IGARSS. Volume: IV, s. 41-44 DOI: 10.1109/IGARSS.2009.5417600 (2009)

4. F.A. Trusdell, Mauna Loa - History, Hazards, and Risk of Living With the World's Largest Volcano (USGS Fact Sheet 2012-3104, 2012)

5. F. Amelung, S. Yun, S., T. Walter, P. Segall, S. Kim, Science 316(5827), 1026-1030 (2007)

6. R. Tilling, C. Heliker, D.A. Swanson, D.A., Eruption of Hawaiian Volcanoes - Past, Present, and Future (U.S. Geological Survey General Information, 2010)

7. T. Farr, P. Rosen, E. Caro, R. Crippen, R. Duren, S. Hensley, M. Kobrick, M. Paller, E. Rodriguez, L. Roth, D. Seal, S. Sha_er, J. Shimada, J. Umland, M. Werner, M. Oskin, D. Burbank, D. Alsdorf, Reviews of Geophysics, 45 (2007)

8. P. Berardion, IEEE Transactions on geoscience and remote sensing, Volume 40, Issue 11, 2375-2382 (2002) 